\title{
Simultaneous and Gradient IPN of Polyurethane/Vinyl Ester Resin: Morphology and Mechanical Properties
}

\author{
Dongyan Tang, ${ }^{1}$ Xiaohong Zhang, ${ }^{2}$ Lili Liu, ${ }^{1}$ and Liangsheng Qiang1 \\ ${ }^{1}$ Department of Chemistry, School of Science, Harbin Institute of Technology, Harbin 150001, China \\ ${ }^{2}$ College of Materials Science and Chemical Engineering, Harbin Engineering University, Harbin 150001, China
}

Correspondence should be addressed to Dongyan Tang, tdybox@yahoo.com.cn

Received 14 February 2009; Revised 21 June 2009; Accepted 28 October 2009

Recommended by Xiaogong Wang

A series of polyurethane (PU) and vinyl ester resin (VER) simultaneous and gradient interpenetrating polymer networks (represented as s-IPN and g-IPN, resp.) curing at room temperature were prepared by changing the component ratios of PU or VER in s-IPN, time intervals, and component ratio sequences of s-IPN in g-IPN. The microstructures of s-IPN and g-IPN were detected by atomic force microscope (AFM), dynamic mechanical analyzer (DMA), and surface constitution scanning of nitrogen element of energy dispersive X-ray spectrum (EDX), respectively. The mechanical properties of s-IPN and g-IPN were studied by values in strain-stress curves detected by electronic multipurpose tester. The results indicated that the morphology and mechanical properties are both affected by PU/VER component ratios in s-IPN, gradient time intervals, and gradient component ratio sequences. Furthermore, the morphology detection by EDX and mechanical properties study both proved the formation of gradient structures in transition regions of g-IPN.

Copyright (c) 2009 Dongyan Tang et al. This is an open access article distributed under the Creative Commons Attribution License, which permits unrestricted use, distribution, and reproduction in any medium, provided the original work is properly cited.

\section{Introduction}

Interpenetrating polymer networks (IPNs) are a special class of polymer alloys synthesized with two or more distinct crosslinked polymer networks held together by permanent entanglement $[1,2]$. They can exhibit much higher mechanical properties than that of the constituent polymers and that of ordinary multicomponent systems $[3,4]$ due to their interpenetrating structures created between two networks. And gradient IPN (g-IPN) is mixture of crosslinked polymers in which the concentration of one network of IPN changes across the section of a sample, and thus the physical or chemical properties also change across the section of a sample. They can be obtained by changing the concentration of one network of simultaneous IPN (s-IPN) across the section of a sample gradually and continuously. Thus, gIPN $[5,6]$ can be regarded as a combination of an infinite number of layers of simultaneous IPN and show much more interpenetrating structures, resulting in different properties from the individual networks and IPN [7-10]. Enormous efforts have been focused, in both academic and industrial laboratories, on the preparation of g-IPN with excellent physical and chemical properties, and, however, reports on its properties of g-IPN are still limited [11] due to the complexity of preparation for multicomposition system and room temperature curing system of gradient IPN. Moreover, there are few reports on the control, verify, and estimate [12] of the gradient structures in multicomponent and multiphase IPN systems which exhibit great importance for the design of novel materials with unexpected improvements in mechanical and/or other properties.

In our present papers, a series of polyurethane/vinyl ester resin (PU/VER) simultaneous and gradient IPN curing at room temperature are successfully synthesized by changing the component ratios of PU or VER in IPN and casting the mixture with different component ratios in a mold at various times and by different component ratio sequences. The relationships of morphology of IPN and g-IPN with mechanical properties are studied by the results obtained by strain-stress curves and microstructure observation and detections. The gradient structures formed in system are proved by the results of energy dispersive $\mathrm{X}$-ray spectrum and nitrogen element surface scanning. 


\section{Experimental}

2.1. Preparation of Simultaneous IPN and Gradient IPN. A weight amount of trimethylol propane (TMP, as crosslinker of PU) was dissolved in dyhydrated ethyl acetate (EAc) first and then 1,4-butylene glycol (1,4-BD, as chain-extender of PU), epoxy acrylate (supplied by Shanghai Xinhua Resin Plant, the main structure is formed by the chain extention of linear acrylate resin with bisphenol A and chloroepoxy propane and then is crosslinked by aromatic diisocyanate), PU prepolymer (toluene diisocyanate precursor, self prepared by using toluene diisocyanate and polyoxypropylene glyco (PPG, WM: about 3000; the hydroxyl value: about 52.0.), and benzoyl peroxide-N,N'-dimentylaniline (BPODMA, as redox initiators of epoxy acrylate) were sequentially added into the above system. After the mixture was thoroughly stirred and degassed under vacuum for 5-10 minutes, a transparent sample was prepared by curing in a mold. A series of simultaneous IPN (s-IPN, represented as $x / y$, which $x$ refers to PU component, $y$ to VER component) were synthesized by varying the component ratios of PU and VER in the system. The g-IPN was prepared by casting the mixture of different component ratios in a mold sequentially. A series of g-IPN were obtained by changing the time intervals and component sequences (represented as $x_{1} / y_{1} \sim x_{2} / y_{2} \sim$ $x_{3} / y_{3}$, each $x / y$ represents each component ratio of s-IPN in g-IPN) during the curing process of s-IPN.

2.2. Measurements. Tensile strength $(\sigma)$, elongation at break $(\varepsilon)$, and elastic modulus $(E)$ of s-IPN and g-IPN of small size dumb-bells were measured from strain-stress curves by Zwick Roell Z1010 testing machine at $25^{\circ} \mathrm{C}$ with a crosshead speed of $2 \mathrm{~mm} / \mathrm{min}$ according to GB4456-84. Data were taken as average of at least five measurements. Atomic force microscopy (AFM) imaging was performed on microtomed surfaces with a digital instrument nanoscope III scanning probe microscope with multimode head and Jscanner. The tapping mode was used at ambient conditions. The resonance frequencies of these probes were in the 300 $\mathrm{KHz}$ range. Phase images of two dimensions were recorded simultaneously. Dynamic mechanical analyzer (DMA) measurements were made at $100 \mathrm{~Hz}$ in the temperature range of $-75 \sim 150^{\circ} \mathrm{C}$ with a heating rate of $10^{\circ} \mathrm{C} / \mathrm{min}$. Energy dispersive X-ray (EDX) analysis was performed using Oxford ISIS-300 spectrometer coupled to a JEOL JXA-scanning electron microscope on the cross-section of a sample.

\section{Results and Discussion}

\subsection{Mechanical Properties Study}

3.1.1. Mechanical Properties of s-IPN with Different Component Ratios. Generally, s-IPN shows better elongation at break and tensile strength than those of ordinary polymers and other types of multicomponent systems due to the mutual entanglement, forced compatibility, and synergism of the networks in s-IPN. In the s-IPN of PU/VER, VER is the hard segment contents. Thus, both the values of elongation at break and tensile strength are proportional to the VER

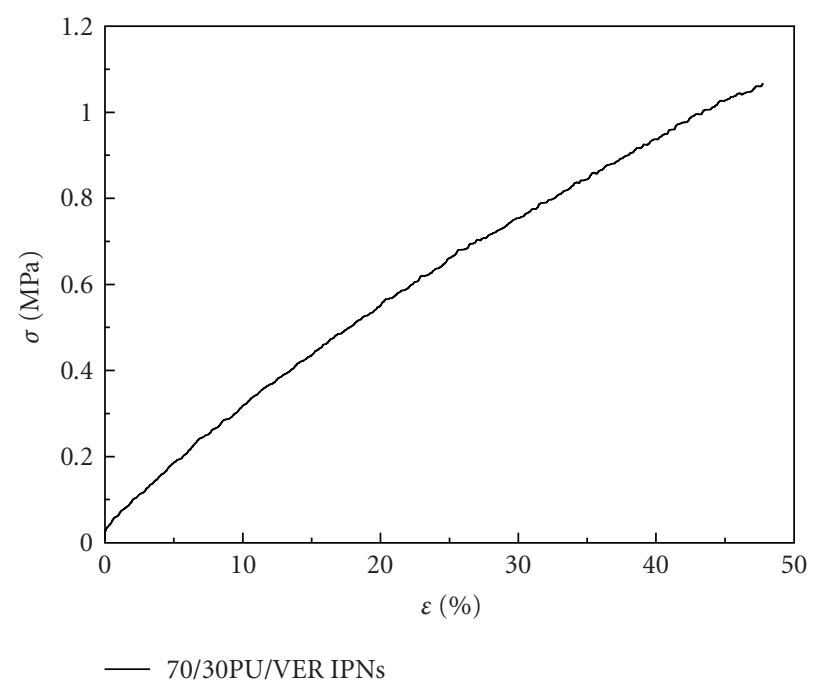

FIGURE 1: Strain-stress curves of s-IPN with different component ratios.

content in s-IPN. Figure 1 shows the strain-stress curves of 70/30 s-IPN. As shown in Figure 1, the s-IPN shows the mechanical properties of PU mostly, because of the higher elastic properties of PU and more component ratios in s-IPN. It is suggested that the thermodynamic miscibility created by the amino group in VER and the isocyanate group in PU lead to approximate compatibility of s-IPN [13].

3.1.2. Mechanical Properties of g-IPN with Different Component Sequences and Time Intervals. Similar to the effects of VER in S-IPN to mechanical properties, higher modulus of VER in g-IPN also contributes better mechanical properties to g-IPN. Different component ratio of PU and VER results in the different modulus contributions of PU and VER networks in system. The different component sequence of PU to VER networks with different component ratio is another factor to affect mechanical properties of g-IPN. Figure 2 shows the strain-stress curves of g-IPN at different time intervals with different component sequences. Seen from the figure, two g-IPNs show approximate the same values of elongation at break. But more VER contents in s-IPN in each sequence give apparent higher tensile strengths values. Furthermore, the results listed in Table 1 are calculated from the stress-strain curves. The results listed in Table 1 indicate that the gradient techniques can do improve the elastic properties of IPN greatly. The g-IPN in different components sequences with higher content of VER exhibits the higher tensile strength values and lower elongation values. Especially for the g-IPN with 70/30 $80 / 20 \sim 90 / 10$ components sequences, it shows higher elongation at break values without a dramatic decrease in the tensile strength, demonstrating the formation of gradient structures between different s-IPN. It provides a basis for the design of novel materials with unexpected improvements in elastic properties and appropriate tensile strength.

Figure 3 shows the strain-stress curves of 70/30 $80 / 20 \sim 90 / 10$ g-IPN with different time intervals. It can be 


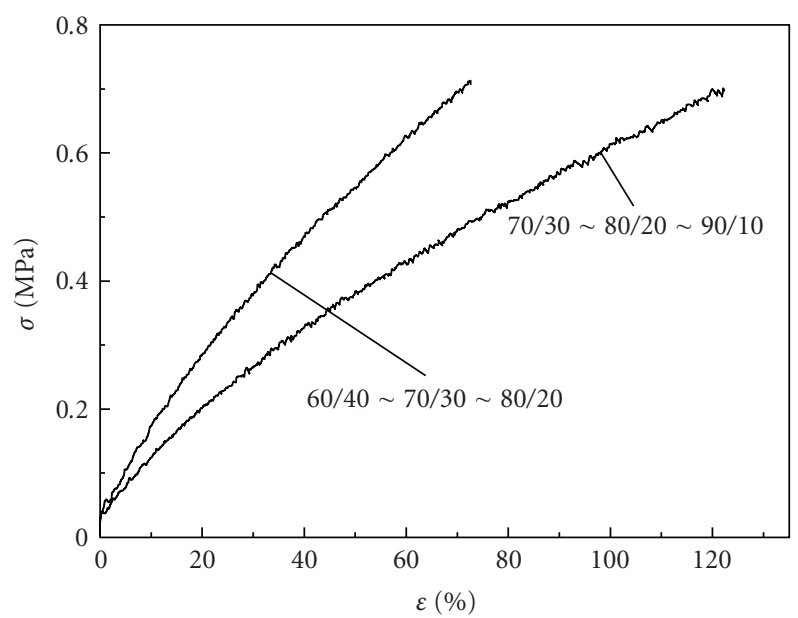

(a)

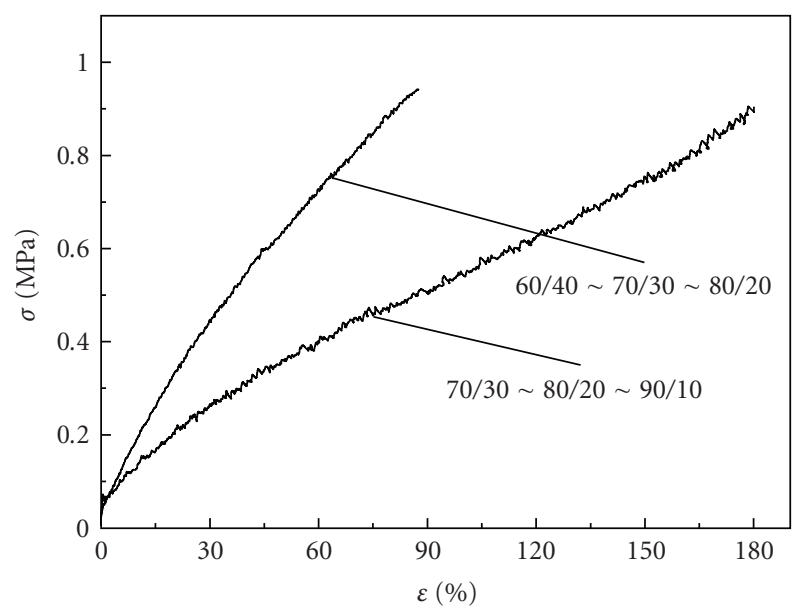

(b)

Figure 2: Strain-stress curves of g-IPN with different component sequences at different time intervals: (a) $t=2.0$ hours and (b) $t=$ 3.5 hours.

TABLE 1: Mechanical properties calculation results of 70/30 s-IPN and g-IPN with different component sequences.

\begin{tabular}{lcccc}
\hline Samples & $t / \mathrm{h}$ & $E / \mathrm{MPa}$ & $\sigma / \mathrm{MPa}$ & $\varepsilon / \%$ \\
\hline \multirow{3}{*}{$70 / 30 \sim 80 / 20 \sim 90 / 10$ g-IPN } & 2 & 0.8 & 0.70 & 126.94 \\
& 3 & 0.7 & 0.74 & 140.16 \\
& 3.5 & 0.6 & 0.91 & 193.78 \\
\hline \multirow{3}{*}{$60 / 40 \sim 70 / 30 \sim 80 / 20$ g-IPN } & 2 & 1.3 & 0.71 & 75.00 \\
& 3 & 1.2 & 0.83 & 101.78 \\
& 3.5 & 1.6 & 0.94 & 89.69 \\
\hline 70/30 s-IPN & & 2.9 & 1.07 & 49.09 \\
\hline
\end{tabular}

observed that the partially gradient structures in transitional regions between the layers for sample with 3.5-hour intervals can contribute better mechanical properties. The calculation results from the strain-stress curves are listed in Table 2. All g-IPN show higher elongation at break than those of sIPN, proving the synergistic action of more interpenetrating effects of g-IPN.

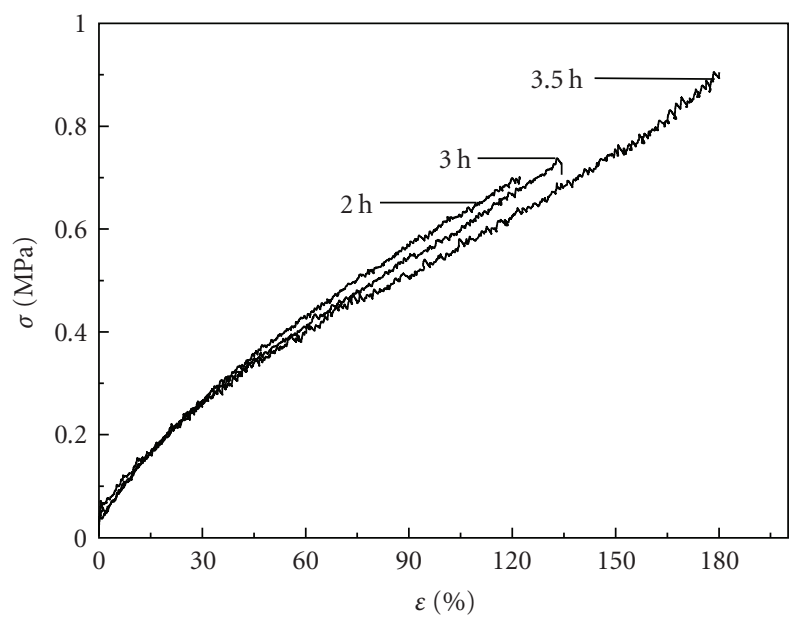

FIGURE 3: Strain-stress curves of g-IPN with different time intervals.

TABle 2: Mechanical properties calculation results of $60 / 40 \sim$ $70 / 30 \sim 80 / 20$ g-IPN with different time intervals.

\begin{tabular}{lccc}
\hline$t / \mathrm{h}$ & $E / \mathrm{MPa}$ & $\sigma / \mathrm{MPa}$ & $\varepsilon / \%$ \\
\hline 2 & 0.8 & 0.70 & 126.94 \\
3 & 0.7 & 0.74 & 140.16 \\
3.5 & 0.6 & 0.91 & 193.78 \\
\hline
\end{tabular}

\subsection{Morphology Study}

3.2.1. Morphology of s-IPN with Different Component Ratios. The mechanism of curing reactions for PU and VER is different, and thus the two networks could be formed simultaneously. In most cases of the formation of IPN, to improve the thermodynamic compatibility between two different components, researchers often introduce the graft structures between two networks. In this paper, the amino group in VER can react with the isocyanate group in PU to form the graft-IPN. Figure 4 gives the AFM (phase images of two dimensions) photographs of s-IPN with different component ratios.

The bright phase is the PU phase with lower modulus and the darker phase is the VER phase with higher modulus. As shown in Figure 4, the dispersed VER phase is also linked and the continuous degree increases with the PU component ratios in system. The results of AFM indicate that the phase ranges of PU/VER s-IPN obtained are all in nanometer scales owing to the possible graft structures formed. Similarly, the compatibility of $80 / 20 \mathrm{~s}$-IPN is better than that of $70 / 30$ and 90/10 s-IPN because of the smaller domain sizes in AFM photos.

The morphology of IPN also can be studied further by DMA detection. From the loss factor $(\tan \delta)$ versus temperature curve of DMA, the degree of inward shift of $T_{g}$ of s-IPN, compared with that of pure PU and pure VER, and the numbers of $T_{g}$ peaks in $\tan \delta \sim T$ of both can indicate the degree of phase mixing in each component [14]. Figure 5 shows the plots of loss factor $(\tan \delta)$ versus 


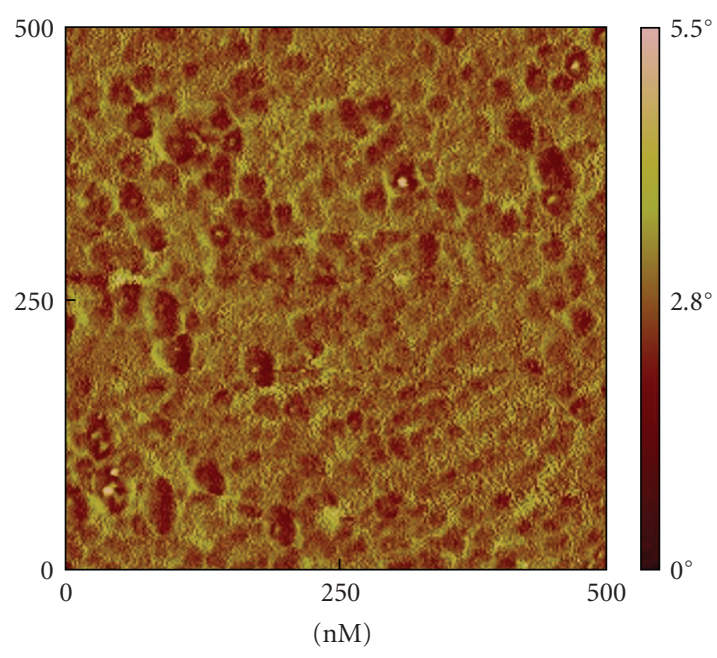

(a)

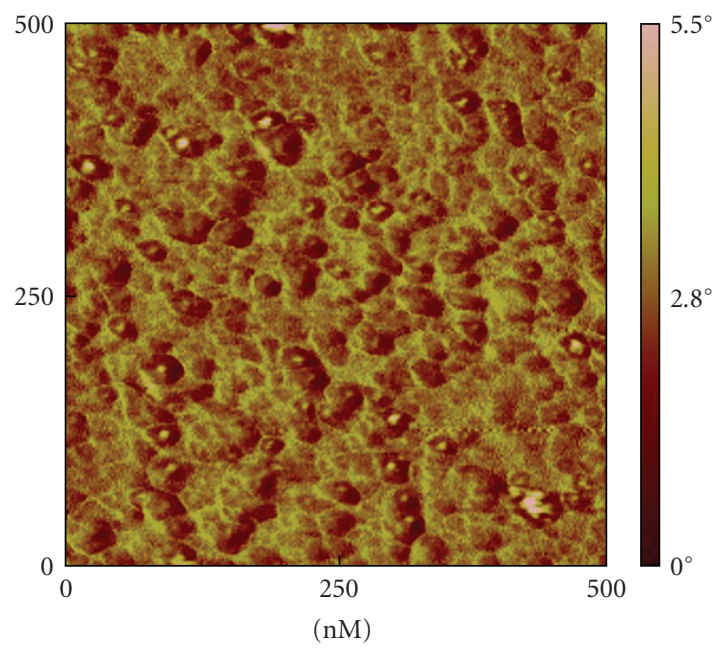

(b)

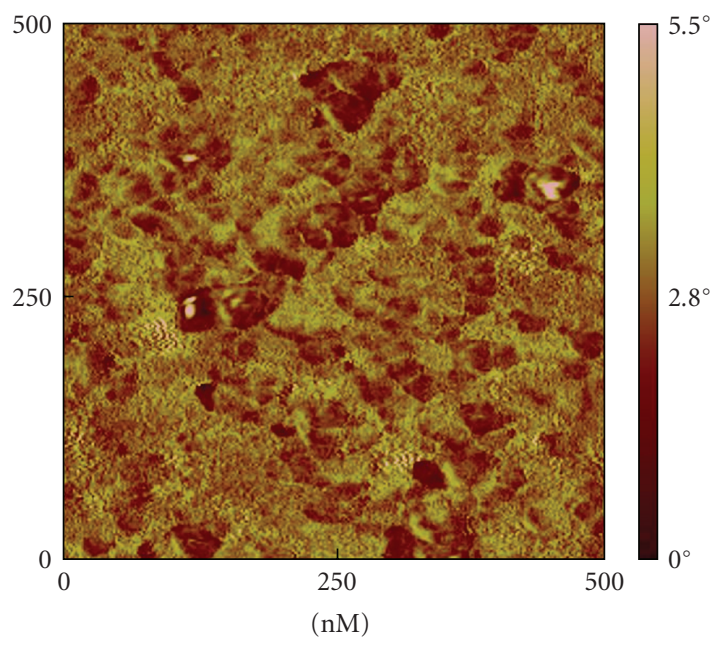

(c)

FIGURE 4: AFM photographs of s-IPN: (a) 90/10; (b) 80/20; (c) $70 / 30$ s-IPN.

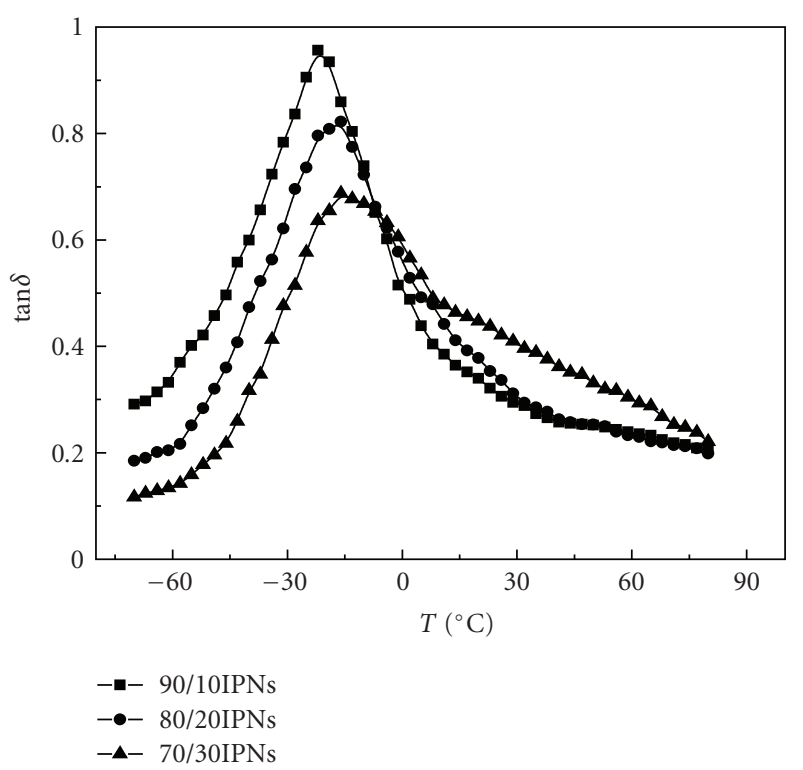

FIGURE 5: Loss factor versus temperature curves of IPN with different component ratios.

temperature of various PU/VER component ratios of sIPN. As shown in the figure, the PU/VER component ratio affects morphology of IPN. The maximum value of $\tan \delta$ shifts toward lower temperatures. These results can be attributed to the fact that PU phase in 70/30 90/10 IPN polymerizes first and forms the continuous phase. The results also indicate that multicomponent system can broaden the effective $T_{g}$ range and move the range toward higher regions corresponding to higher VER content. All the s-IPNs show one expended $T_{g}$ peaks with maximum $\tan \delta$ value higher than 0.4 in near $50^{\circ} \mathrm{C}$ temperature ranges. These show better interpenetration and apparent entanglement effects led by better compatibility between two components of s-IPN. Meanwhile, there appear relatively expended ranges in $\tan \delta \sim T$ curves. According to the Rouse-Bueche theory, such single broad peaks do not mean homogeneous system formed.

3.2.2. Morphology of g-IPN with Different Components Sequences. Figure 6 shows the plots of loss factor $(\tan \delta)$ versus temperature of the g-IPN with different components ratio sequences $(60 / 40 \sim 70 / 30 \sim 80 / 20 \mathrm{IPN}$ and 70/30 $80 / 20 \sim 90 / 10$ IPN). Compared with s-IPN, the broad damping plateaus with higher $\tan \delta$ is the result of superposition of a great number of relaxation maxima in the layer and transition regions between layers with progressively varying compositions. The better $\tan \delta$ ranges are higher than 0.4 in near $100^{\circ} \mathrm{C}$ temperature ranges. The morphology study of $\mathrm{g}$-IPN is consistent with the result of mechanical properties detections shown in Figures 2 and 3.

3.2.3. Verifying the Gradient Structures of g-IPN. In the present study, considering that g-IPNs contain nitrogen, and its content contributes by PU network, the detection of the 


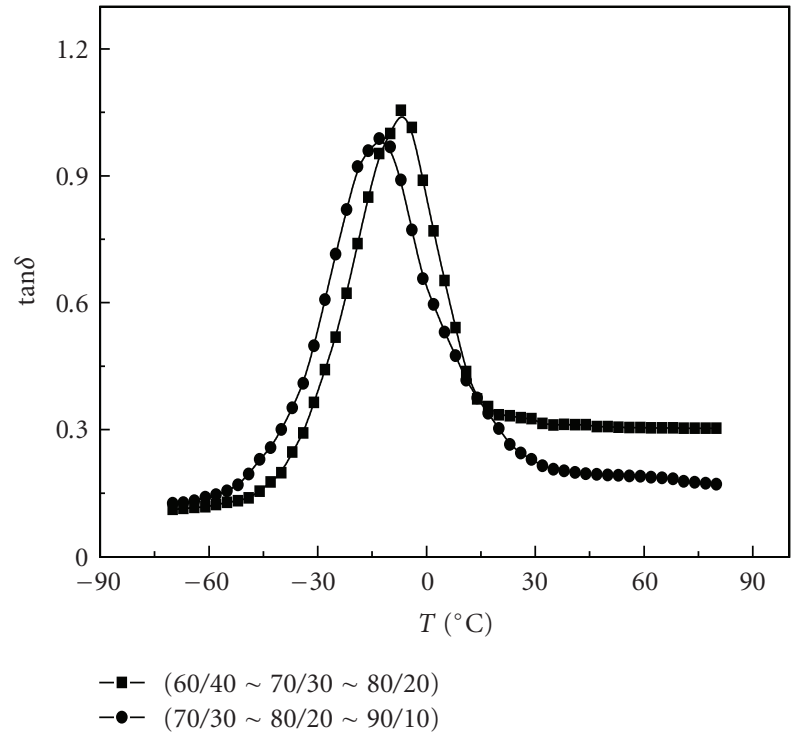

Figure 6: Loss factor versus temperature curves of the g-IPN with different component sequences.

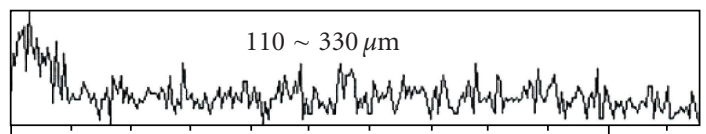

(a)

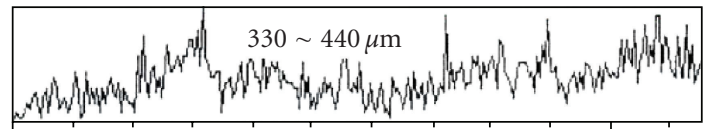

(b)

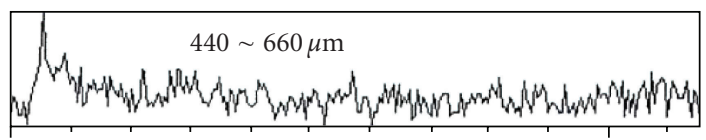

(c)

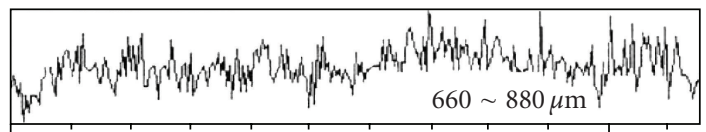

(d)

FIGURE 7: The surface line scanning of element $\mathrm{N}$ on the different cross section of g-IPN.

content changing of $\mathrm{N}$ element along the samples in system can illustrate the changing of PU in g-IPN. Figure 7 gives the surface line scanning of element $\mathrm{N}$ on the cross section of gIPN with the component ratios of 70/30 80/20 90/10. Figure 8 gives the surface constitution scanning of element $\mathrm{N}$ on the cross section of transitional regions of g-IPN. Both the EDX analysis of g-IPN in Figures 7 and 8 verify the gradient structures of g-IPN through the nitrogen element distribution detection in the layer $(220 \sim 440 \mu \mathrm{m})$ and in the transition regions between layers $(440 \mu \mathrm{m} \sim 550 \mu \mathrm{m})$. The element $\mathrm{N}$ is homogeneously distributed in the layer, while

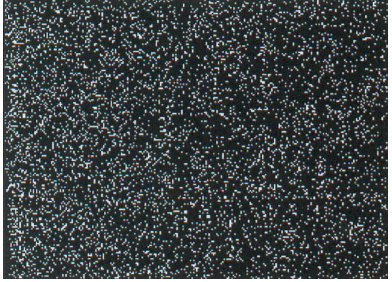

(a)

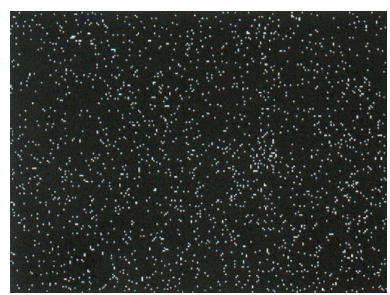

(b)
Figure 8: The nitrogen element constitution of surface scans of gIPN at different parts of layer: (a) $220 \mu \mathrm{m} \sim 440 \mu \mathrm{m}$; (b) $440 \mu \mathrm{m} \sim$ $550 \mu \mathrm{m}$.

the $\mathrm{N}$ distribution in the transition regions varies gradually from one layer to another layer.

\section{Conclusion}

A series of simultaneous and gradient IPN with different miscibility and mechanical properties are prepared by varying the component ratios of PU to VER in simultaneous IPN, time intervals, and component ratio sequences in gradient IPN. The elastic properties of IPN can be improved evidently by interpenetrating techniques and gradient structures formed in transitional regions. PU/VER component ratios in s-IPN, time intervals, and component ratio sequences in g-IPN all affect the morphology. The formation of gradient constructions in transition regions of g-IPN can be proved by morphology studies through EDX analysis and mechanical properties detections. The content of nitrogen in g-IPN changes with different trend along the direction of sample. And the morphology observation and detection results are both consistent with the results gained by mechanical detections.

\section{Acknowledgments}

The authors gratefully acknowledge the National Natural Science Foundation of China under Grant no. 50675045, the Program for New Century Excellent Talents in University (NCET-08-0165), and Youth Science and Technology Foundation of Heilongiiang Province, China (QC07C01).

\section{References}

[1] D. J. Hourston and F.-U. Schäfer, "Poly(ether urethane)/poly(ethyl methacrylate) IPNs with high damping characteristics: the influence of the crosslink density in both networks," Journal of Applied Polymer Science, vol. 62, no. 12, pp. 2025-2037, 1996.

[2] D. Y. Tang, L. S. Qiang, and Z. Jin, "Preparation, morphology, and thermoelectric property studies of $\mathrm{BaTiO}_{3}$ superfine fiber/castor oil polyurethane-based IPN nanocomposites," Journal of Applied Polymer Science, vol. 84, no. 4, pp. 709-715, 2002.

[3] K. H. Hsieh, J. L. Han, C. T. Yu, and S. C. Fu, "Graft interpenetrating polymer networks of urethane-modified bismaleimide 
and epoxy (I): mechanical behavior and morphology," Polymer, vol. 42, no. 6, pp. 2491-2500, 2001.

[4] F. J. Hua and C. P. Hu, "Interpenetrating polymer networks of epoxy resin and urethane acrylate resin: 2. Morphology and mechanical property," European Polymer Journal, vol. 36, no. 1, pp. 27-33, 1999.

[5] X. Cheng, Z. Chen, T. Shi, and H. Wang, "Synthesis and characterization of core-shell LIPN-fluorine-containing polyacrylate latex," Colloids and Surfaces A, vol. 292, no. 2-3, pp. 119-124, 2007.

[6] S. Murayama, S.-I. Kuroda, and Z. Osawa, "Hydrophobic and hydrophilic interpenetrating polymer networks (IPNs) composed of polystyrene and poly(2-hydroxyethyl methacrylate): 2. Gradient composition in the IPNs synthesized by photopolymerization," Polymer, vol. 34, no. 18, pp. 38933898, 1993.

[7] P. Pissis, G. Georgoussis, V. A. Bershtein, E. Neagu, and A. M. Fainleib, "Dielectric studies in homogeneous and heterogeneous polyurethane/polycyanurate interpenetrating polymer networks," Journal of Non-Crystalline Solids, vol. 305, no. 1-3, pp. 150-158, 2002.

[8] L. Karabanova, L. Sergeeva, and G. Boiteux, "Filler effect on formation and properties of reinforced interpenetrating polymer networks," Composite Interfaces, vol. 8, no. 3-4, pp. 207-219, 2001.

[9] Y.-S. Kim and S.-C. Kim, "Properties of polyetherimide/dicyanate semi-interpenetrating polymer network having the morphology spectrum," Macromolecules, vol. 32, no. 7, pp. 2334-2341, 1999.

[10] K. O. Sylvester-Hvid, S. Rettrup, and M. A. Ratner, "Twodimensional model for polymer-based photovoltaic cells: numerical simulations of morphology effects," Journal of Physical Chemistry B, vol. 108, no. 14, pp. 4296-4307, 2004.

[11] D. Tang, C. Qin, W. Cai, and L. Zhao, "Preparation, morphology, and mechanical properties of modified-PU/UPR graftIPN nanocomposites with $\mathrm{BaTiO}_{3}$ fiber," Materials Chemistry and Physics, vol. 82, no. 1, pp. 73-77, 2003.

[12] I. Karabanova, P. Pissis, A. Kanapitsas, and E. Lutsyk, "Thermodynamic state, temperature transitions, and broadband dielectric relaxation behavior in gradient interpenetrating polymer networks," Journal of Applied Polymer Science, vol. 68, no. 1, pp. 161-171, 1998.

[13] D. Tang, J. Zhang, D. Zhou, and L. Zhao, "Influence of $\mathrm{BaTiO}_{3}$ on damping and dielectric properties of filled polyurethane/unsaturated polyester resin interpenetrating polymer networks," Journal of Materials Science, vol. 40, no. 13, pp. 3339-3345, 2005.

[14] J. Yang, M. A. Winnik, D. Ylitalo, and R. J. DeVoe, "Polyurethane-polyacrylate interpenetrating networks. 1. Preparation and morphology," Macromolecules, vol. 29, no. 22, pp. 7047-7054, 1996. 

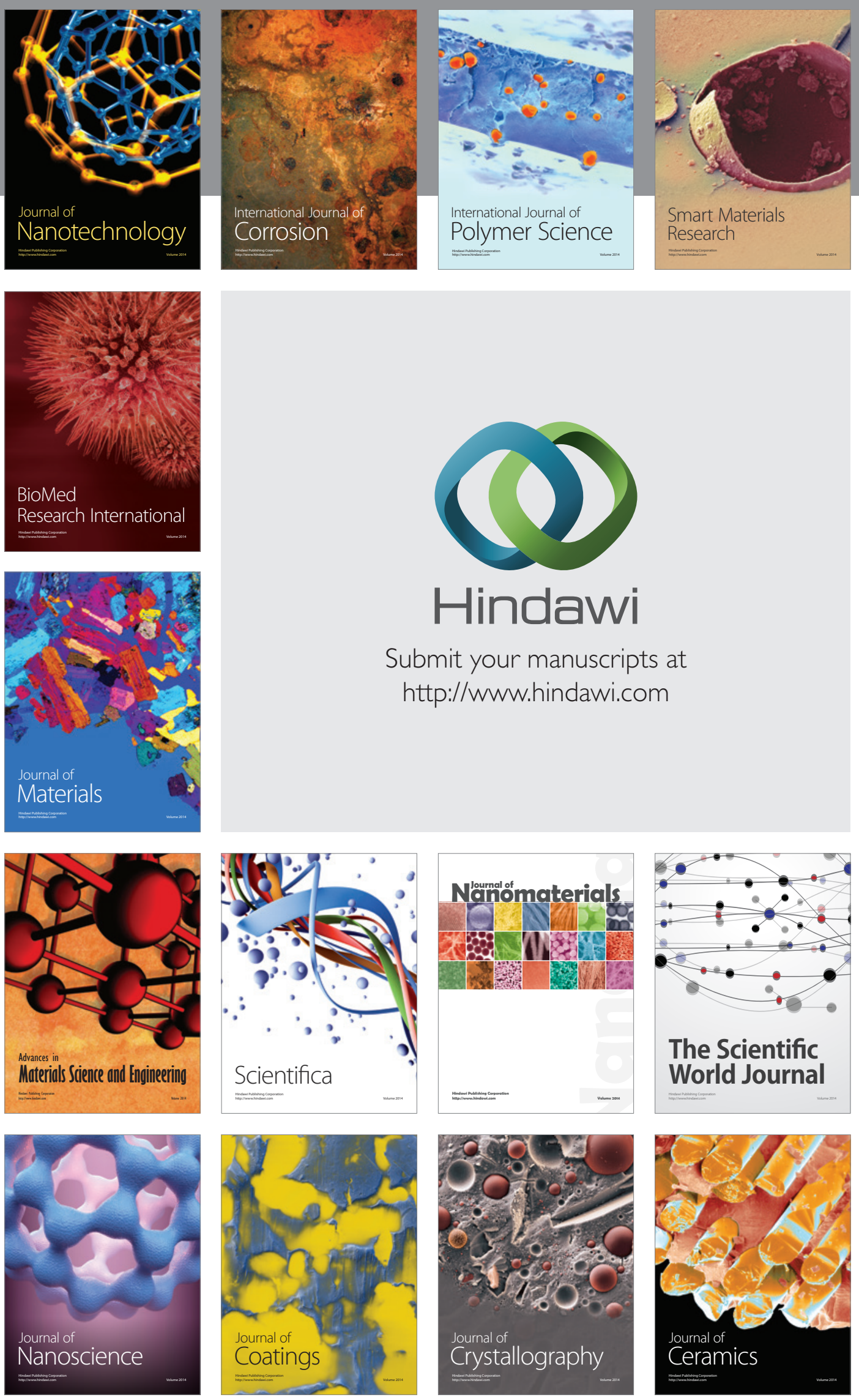

The Scientific World Journal

Submit your manuscripts at

http://www.hindawi.com

\section{World Journal}

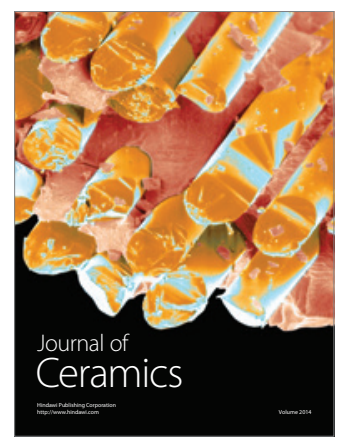

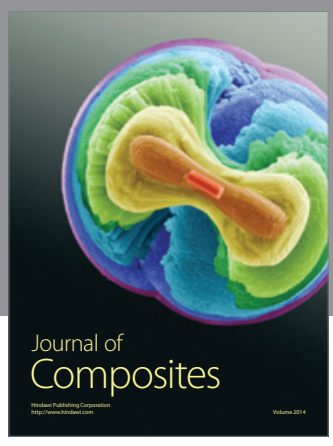
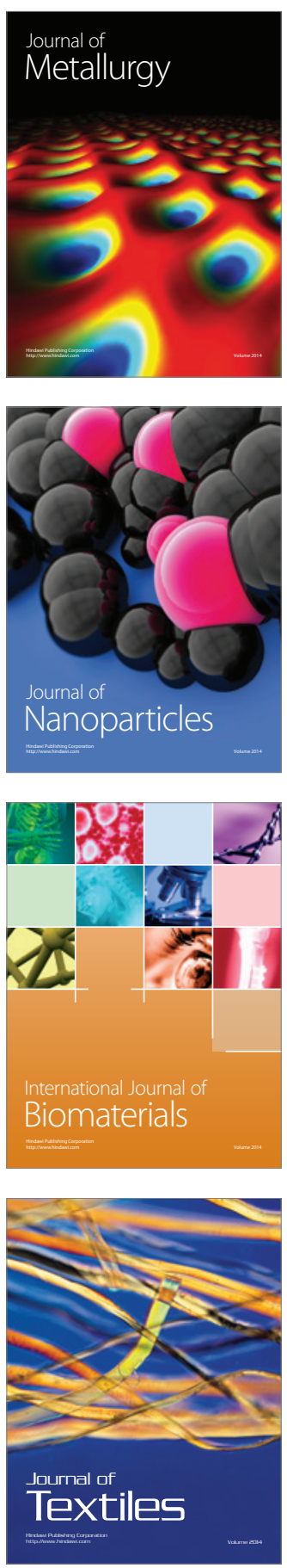